$d^{\prime \prime}$ as close as we please to $d$. This completes the proof that $S$ has property (iii).

\title{
BIBLIOGRAPHY
}

1. P. Koebe, Zur konformen Abbildung unendlich-vielfach zusammenhängender schlichter Bereiche auf Schlitzberciche, Nachr. Kgl. Ges. Wiss. Göttingen Math.-Phys. Kl. (1918), 60-71.

2. E. Reich, A counterexample of Koebe's for slit mappings, Proc. Amer. Math. Soc. 11 (1960), 970-975.

WASHINGTON UNIVERSITY

\section{IDEALS OF SQUARE SUMMABLE POWER SERIES}

\section{JAMES ROVNYAK}

Let $\mathfrak{C}$ be a Hilbert space. By $\mathfrak{C}(z)$ we mean the Hilbert space of all formal power series $f(z)=\sum a_{n} z^{n}$ in the indeterminate $z$ with coefficients $a_{n}$ in $\mathfrak{C}$, such that

$$
\|f\|^{2}=\sum\left\|a_{n}\right\|^{2}<\infty .
$$

Although $\mathfrak{e}(z)$ may be thought of as a space of $\mathfrak{C}$-valued functions analytic in the unit disc, we prefer the point of view adopted above in which the notation $\mathcal{C}(z)$ is used as the algebraist uses $K[x]$ for the ring of polynomials in $x$ over a field $K$.

An ideal of $\mathfrak{C}(z)$ is a vector subspace $\mathfrak{T}$ of $\mathfrak{C}(z)$ which contains $z f(z)$ whenever it contains $f(z)$. We will obtain the structure of the closed ideals of $\mathfrak{C}(z)$. This problem was solved in [6] when $\mathfrak{C}$ has dimension 1 , and in this form may be regarded as an interpretation of the work of Beurling [1]. One advantage of our formulation is that it generalizes naturally to Hilbert spaces $\mathfrak{C}$ of arbitrary dimension.

The solution is in terms of formal power series $B(z)=\sum B_{n} z^{n}$ whose coefficients $B_{n}$ are bounded linear transformations (i.e., operators) on e. We write $N=N(B)$ for the set of all $c$ in $\mathcal{e}$ such that $B(z) c=\sum B_{n} c z^{n}$ vanishes identically; in other words, $N$ is the intersection of the null spaces of the operators $B_{n}$. The series $B(z)$ of interest are those which satisfy (1), whenever $\left(c_{n}\right)$ is a sequence of unit vectors in $\mathfrak{C}$ orthogonal to $N,\left(z^{n} B(z) c_{n}\right)$ is an orthonormal set in $\mathfrak{C}(z)$. When (1) is satisfied we write $\operatorname{Tr}(B)$ for the set of all formal products $B(z) f(z)$ with $f(z)$ in

\footnotetext{
Received by the editors January 21, 1961 and, in revised form, April 3, 1961.
} 
$\mathcal{C}(z)$. Any $g(z)$ in $\mathfrak{T}(B)$ may be written $g(z)=B(z) f(z)$ where the coefficients of $f(z)$ are orthogonal to $N$, and the proof of Lemma 9 of de Branges [2] shows that $\|g\|=\|f\|$. Hence it is clear that $\operatorname{mr}(B)$ is a closed ideal of $\mathfrak{e}(z)$.

TheOREM. If $\mathfrak{T}$ is any closed ideal of $\mathfrak{e}(z)$, there is formal power series $B(z)$ with operator coefficients which satisfies (1), such that $\mathfrak{N}=\mathfrak{M}(B)$.

LEMмa 1. Let $B(z)$ be a formal power series with operator coefficients which satisfies (1). If $f(z)$ is in $\mathrm{C}(z)$ and has coefficients orthogonal to $N(B)$, the product $B(z) f(z)$ is in $\mathfrak{C}(z)$ and has the same norm as $f(z)$.

LeMмa 2. If e has finite dimension $r$ and contains $c_{1}, \cdots, c_{r}$ as an orthonormal basis, then

$$
\sum \operatorname{sgn} \alpha\left(a_{\alpha(1)}, c_{1}\right) \cdots\left(a_{\alpha(r)}, c_{r}\right) a_{\alpha(r+1)}=0
$$

for any $r+1$ elements $a_{1}, \cdots, a_{r}$ of $\mathfrak{C}$. Here $\alpha$ ranges in the permutations of $1, \cdots, r+1$ and $\operatorname{sgn} \alpha$ is +1 or -1 according as $\alpha$ is even or odd.

Lemma 3. Let $\mathfrak{e}$ have finite dimension $r$ and let $c_{1}, \cdots, c_{r}$ be an orthonormal basis for $\mathcal{C}$. Let $f_{1}(z), \cdots, f_{r+1}(z)$ be any $r+1$ elements of $\mathcal{e}(z)$ and set $f_{i j}(z)=\sum\left(a_{j n}, c_{i}\right) z^{n}$ if $f_{j}(z)=\sum a_{j n} z^{n}$. Then

$$
\sum \operatorname{sgn} \alpha f_{1, \alpha(1)}(z) \cdots f_{r, \alpha(r)}(z) f_{\alpha(r+1)}(z)=0 .
$$

The zero on the right is the formal power series which vanishes identically, and the sum is taken as in Lemma 2.

Lemma 4. Let $\mathfrak{e}$ have finite dimension $r$ and let $c_{1}, \cdots, c_{r}$ be an orthonormal basis for $\mathcal{C}$. Let $f_{1}(z), \cdots, f_{r}(z)$ be an orthonormal set in $\mathfrak{C}(z)$ such that $z^{m} f_{i}(z)$ is orthogonal to $z^{n} f_{j}(z)$ whenever $m \neq n$, and let $f_{i j}(z)$ be as in Lemma 3. Then

$$
g(z)=\operatorname{det}\left(f_{i j}(z)\right)
$$

is a formal power series with complex coefficients, and when regarded as a formal power series with operator coefficients, $g(z)$ satisfies (1) with $N(g)=0$.

LEMMA 5. If $\mathfrak{e}$ has finite dimension $r$, there is no orthonormal set $f_{1}(z), \cdots, f_{r+1}(z)$ in $\mathrm{e}(z)$ containing $r+1$ elements and such that $z^{m} f_{i}(z)$ is orthogonal to $z^{n} f_{j}(z)$ whenever $m \neq n$.

Lemma 6. Let $\mathfrak{T}$ be a closed ideal of $\mathfrak{C}(z)$ and let $B$ be the orthogonal 
complement in TH of the series $z f(z)$ with $f(z)$ in $\mathbb{T}$. Then there exists a linear isometric transformation $S$ from $B$ to $\mathcal{C}$.

Proof of Lemma 1. This is the same as the proof of Lemma 9 of de Branges [2].

Proof of Lemma 2. By linearity it is sufficient to establish the formula when each $a_{i}$ is equal to some $c_{j}$. In this case two of the $a_{i}$ must be equal. By a permutation we may suppose $a_{r}=a_{r+1}$. Each term on the left of (2) vanishes unless $a_{1}, \cdots, a_{r}$ are distinct. In this case we may suppose that $a_{i}=c_{i}(i=1, \cdots, r)$. Now the only nonvanishing terms are the ones corresponding to the identity permutation and the permutation which interchanges $r$ and $r+1$ leaving the other numbers fixed. Since the first of these is even and the second odd, the sum in (2) reduces to $a_{r+1}-a_{r+1}=0$.

Proof of Lemma 3. By the definition of formal multiplication, it is sufficient to establish the identity (3) when each $f_{i}(z)$ is a polynomial. In this case, the identity follows from Lemma 2 on replacing each series by the corresponding analytic function. Since an analytic function determines its power series uniquely, the identity holds in the formal sense also.

Proof of Lemma 4. This is the only lemma we were unable to prove by purely formal methods. Let $L^{2}(0,2 \pi)$ be the Hilbert space of a.e. equivalence classes of measurable complex-valued functions $f\left(e^{i \theta}\right)$, $0 \leqq \theta<2 \pi$, such that

$$
\|f\|^{2}=(2 \pi)^{-1} \int_{0}^{2 \pi}\left|f\left(e^{i \theta}\right)\right|^{2} d \theta<\infty .
$$

If $f(z)=\sum a_{n} z^{n}$ is a square summable power series with complex coefficients, let $f\left(e^{i \theta}\right)=\sum a_{n} e^{i n \theta}$ be the corresponding element of $L^{2}(0,2 \pi)$. The sum is taken in the metric of $L^{2}(0,2 \pi)$ and $\|f\|^{2}$ $=\sum\left|a_{n}\right|^{2}$ by Parseval's formula for Fourier series.

If $b$ and $c$ are any fixed elements of $\mathcal{e}, a \rightarrow(a, b) c$ defines an operator on $\mathcal{C}$. Hence we may take the formula

$$
B(z)=\left(, c_{1}\right)\left(f_{1}(z)+\cdots+\left(, c_{r}\right) f_{r}(z)\right.
$$

as the definition of a formal power series with operator coefficients. The hypotheses imply that $\left(z^{n} B(z) a_{n}\right)$ is an orthonormal set in $\mathcal{C}(z)$ whenever $\left(a_{n}\right)$ is a sequence of unit vectors in $\mathcal{C}$, and we have the formula

$$
B(z)=\sum_{i, j=1}^{r}\left(, c_{j}\right) f_{i j}(z) c_{i} .
$$


Hence, using triangular brackets for the inner product in $\mathfrak{C}(z)$,

$$
\begin{aligned}
\delta_{m n} & =\left\langle z^{m} B(z) a_{m}, z^{n} B(z) a_{n}\right\rangle \\
& =(2 \pi)^{-1} \sum_{i, j, k=1}^{r}\left(a_{m}, c_{j}\right)\left(c_{k}, a_{n}\right) \int_{0}^{2 \pi} f_{i j}\left(e^{i \theta}\right) \bar{f}_{i k}\left(e^{i \theta}\right) e^{i(m-n) \theta} d \theta .
\end{aligned}
$$

Taking $a_{m}=c_{p}$ and $a_{n}=c_{q}$, we find that

$$
(2 \pi)^{-1} \int_{0}^{2 \pi}\left\{\sum_{i=1}^{r} f_{i p}\left(e^{i \theta}\right) \bar{f}_{i q}\left(e^{i \theta}\right)\right\} e^{i(m-n) \theta} d \theta=\delta_{p q} \delta_{m n} .
$$

If $p$ and $q$ are held fixed we have a determination of the Fourier coefficients of the integrable function

$$
\sum_{i=1}^{r} f_{i p}\left(e^{i \theta}\right) \bar{f}_{i q}\left(e^{i \theta}\right)
$$

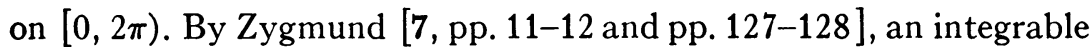
function is essentially uniquely determined by its Fourier series. It follows that

$$
\sum_{i=1}^{r} f_{i p}\left(e^{i \theta}\right) \bar{f}_{i q}\left(e^{i \theta}\right)=\delta_{p q}
$$

a.e. Putting this equation in matrix form we see that

$$
\left|g\left(e^{i \theta}\right)\right|^{2}=\left|\operatorname{det}\left(f_{i j}\left(e^{i \theta}\right)\right)\right|^{2}=1
$$

a.e. Therefore

$$
(2 \pi)^{-1} \int_{0}^{2 \pi}\left|g\left(e^{i \theta}\right)\right|^{2} e^{i(m-n) \theta} d \theta=\delta_{m n}
$$

and it follows that $g(z)$ satisfies (1) with $N(g)=0$.

An elementary proof can be given when $\mathcal{C}$ has dimension 2 . In this case

$$
\begin{aligned}
B(z)= & \left(, c_{1}\right) f_{11}(z) c_{1}+\left(, c_{2}\right) f_{12}(z) c_{1} \\
& +\left(, c_{1}\right) f_{21}(z) c_{2}+\left(, c_{2}\right) f_{22}(z) c_{2}
\end{aligned}
$$

Let

$$
\begin{aligned}
C(z)= & \left(, c_{1}\right) f_{22}(z) c_{1}-\left(, c_{2}\right) f_{12}(z) c_{1} \\
& -\left(, c_{1}\right) f_{21}(z) c_{2}+\left(, c_{2}\right) f_{11}(z) c_{2} .
\end{aligned}
$$

It is easy to see that since $B(z)$ satisfies $(1)$ with $N(B)=0, C(z)$ satisfies (1) with $N(C)=0$. Then $g(z)=B(z) C(z)$ when $g(z)$ is considered 
as a formal power series with operator coefficients. Hence, $g(z)$ satisfies (1) with $N(g)=0$ because it is a product of factors with kernels zero. We are unable to give a proof of this sort when $\mathfrak{C}$ has dimension 3.

Proof of Lemma 5. Suppose, to the contrary, that $f_{1}(z), \cdots, f_{r+1}(z)$ is such a set, and write the identity (3) in the form

$$
F_{1}(z) f_{1}(z)+\cdots+F_{r+1}(z) f_{r+1}(z)=0 .
$$

Here $F_{1}(z), \cdots, F_{r+1}(z)$ are formal power series with complex coefficients, and may be regarded as formal power series with operator coefficients. Then by Lemma 4 each $F_{i}(z)$ satisfies (1) with $N\left(F_{i}\right)=0$. By Lemma 1 , each product $F_{i}(z) f_{i}(z)$ is in $\mathrm{e}(z)$ and

$$
\left\langle F_{i}(z) f_{i}(z), F_{j}(z) f_{j}(z)\right\rangle=\left\langle f_{i}(z), f_{j}(z)\right\rangle=\delta_{i j}
$$

at least when $i=j$. To see that the same formula holds for $i \neq j$, write $F_{i}(z)=\sum F_{i n} z^{n}$ and note that

$$
F_{i}(z) f_{i}(z)=\sum F_{i n} z^{n} f_{i}(z)
$$

converges in the metric of $\mathfrak{C}(z)$ since $\left(z^{n} f_{i}(z)\right)$ is an orthonormal set in $\mathcal{C}(z)$ by hypothesis. Formula (5) now follows from the assumption that $z^{m} f_{i}(z)$ is orthogonal to $z^{n} f_{j}(z)$ whenever $m \neq n$, or $i \neq j$. This completes the proof of (5) and a contradiction is now immediate. For, (5) implies that the left-hand side of (4) has norm $r+1>0$.

PRoOF of Lemma 6. If $\left(c_{\alpha}\right)$ is an orthonormal basis for $\mathcal{e},\left(c_{\alpha} z^{n}\right)$ is an orthonormal basis for $\mathrm{e}(z)$. It is understood that $\alpha$ ranges in some fixed indexing set $A$ and that $n$ ranges in the set $N$ of non-negative integers. The dimension of $\mathfrak{C}$ is the cardinality of $A$ and the dimension of $\mathfrak{e}(z)$ is the cardinality of $A \times N$. If the dimension of $\mathfrak{C}$ is infinite, $A$ and $A \times N$ have the same cardinality, and $\mathcal{C}$ and $\mathcal{C}(z)$ have the same dimension. In this case it is clear that $\operatorname{dim} B \leqq \operatorname{dim} \mathcal{C}$ since $B$ is a closed subspace of $\mathfrak{C}(z)$. The same inequality holds when $\mathfrak{C}$ has finite dimension $r$ by Lemma 5 . For, every orthonormal set $\left(f_{i}(z)\right)$ in $B$ has the property that $z^{m} f_{i}(z)$ is orthogonal to $z^{n} f_{j}(z)$ whenever $m \neq n$. The existence of a linear isometric transformation $S$ from $B$ to $\mathfrak{e}$ now follows from Halmos' discussion of dimension in [5, p. 30].

Proof of Theorem. Let $B$ and $S$ be as in Lemma 6 . Let $c$ be any element of $\mathcal{C}$. By the Schwarz inequality, $g(z) \rightarrow(S g(z), c)$ defines a bounded linear functional on $B$. By the Riesz representation of a continuous linear functional on a Hilbert space, there is a unique element $B(z) c$ of $B$ such that

$$
(S g(z), c)=\langle g(z), B(z) c\rangle
$$


for every $g(z)$ in $B$. Letting $c$ range in $\mathfrak{C}$, (6) defines a formal power series $B(z)$ with operator coefficients, and since $S$ is isometric, $\|B(z) c\|^{2}$ $\leqq\|c\|^{2}$. If $B(z)=\sum B_{n} z^{n}$, then $\left\|B_{n}\right\| \leqq 1$ for every $n$. We prove next that if $c=S g(z)$ is in the range of $S$, then $B(z) c=g(z)$. For if $f(z)$ is any element of $B$, since $S$ is isometric,

$$
\langle f(z), B(z) c\rangle=(S f(z), c)=(S f, S g)=\langle f(z), g(z)\rangle .
$$

Since $f(z)$ is arbitrary, $B(z) c=g(z)$. On the other hand, if $c$ is orthogonal to the range of $S$, the same argument shows that $B(z) c=0$. We conclude that $N(B)$ is the orthogonal complement of the range of $S$ in $\mathcal{C}$. By the definition of $B, B(z)$ satisfies (1).

It is immediate that $\mathfrak{N}(B)$ is contained in $\mathfrak{T}$. For, consider any element $g(z)=B(z) f(z)$ of $\mathfrak{T}(B), f(z)=\sum a_{n} z^{n}$. As we have remarked, we may assume that the coefficients $a_{n}$ of $f(z)$ are orthogonal to $N(B)$. Since $\mathscr{T}$ is an ideal containing $B, B(z) a_{n}$ is in $\mathscr{T}$ for every $n$ as is $z^{n} B(z) a_{n}$. Since $\mathfrak{T}$ is a closed vector subspace of $\mathfrak{C}(z), g(z)=\sum z^{n} B(z) a_{n}$ is in $\mathfrak{T}$.

We prove finally that $\mathfrak{M}(B)$ is all of $\mathfrak{M}$ by showing that the orthogonal complement $\mathfrak{N}$ of $\mathfrak{N}(B)$ in $\mathfrak{T}$ is the zero subspace. This will complete the proof since $\mathfrak{T}(B)$ is closed. Let $f(z)$ be any element of $\Re$. Then $f(z)$ is in $\mathfrak{T}$ and is orthogonal to $B$, and hence of the form $f(z)=z f_{1}(z)$ with $f_{1}(z)$ in $\mathfrak{T}$. For every $g(z)$ in $\mathfrak{T l}(B)$,

$$
\left.\left\langle f_{1}(z), g(z)\right\rangle=\left\langle z f_{1}(z), z g(z)\right\rangle=f(z), z g(z)\right\rangle=0
$$

since $\mathfrak{T}(B)$ is an ideal. Since $g(z)$ is arbitrary, $f_{1}(z)$ is in $\mathscr{T}$. Continuing inductively, $f(z)=z^{n} f_{n}(z)$ for every $n=1,2,3, \cdots$ where $f_{n}(z)$ belongs to $\mathfrak{T}$. It follows that $f(z)$ vanishes identically. Hence $\Re=0$ and $\mathfrak{T}$ is equal to $\mathfrak{T}(B)$.

\section{REFERENCES}

1. A. Beurling, On two problems concerning linear transformations in Hilbert space, Acta Math. 81 (1949), 239-255.

2. L. de Branges, Some Hilbert spaces of entire functions, Trans. Amer. Math. Soc. 96 (1960), 259-295.

3. P. R. Halmos, Finite dimensional vector spaces, Van Nostrand, New York, 1958.

4. - Naive set theory, Van Nostrand, New York, 1960.

5. - Introduction to Hilbert space and spectral multiplicity, Chelsea, New York, 1951.

6. J. Rovnyak, Ideals of square summable power series, Math. Mag. 33 (1960), 265-270 and 34 (1961), 41-42.

7. A. Zygmund, Trigonometric series, Vol. 1, Cambridge, New York, 1959.

YALE UNIVERSITY 